\title{
Minimally Invasive Strabismus Surgery
}

\author{
Daniel S Mojon
}

Head, Department of Strabismology and Neuro-ophthalmology, Kantonsspital St Gallen, University of Bern

\begin{abstract}
In strabismus surgery, the post-operative visibility of the procedure and level of patient discomfort will mainly be influenced by the number and type of muscles operated on and the type and location of the conjunctival openings. For strabismus surgeries minimising tissue disruption, the term minimally invasive strabismus surgery (MISS) has been introduced. MISS uses several keyhole openings (instead of one large muscle access), which are placed where the main surgical steps will be performed. If necessary, tunnels are created between the cuts for some surgical steps. The openings are always placed far away from the limbus. Post-operatively, such cuts will minimise the risk of corneal complications and remain covered by the eyelids. There is increasing evidence to suggest that the disruption of the perilimbal episcleral vessels, which occurs with a limbal incision, may predispose to anterior segment ischaemia. MISS will preserve the majority of perilimbal episcleral vessels. Since MISS also significantly reduces the anatomical disruption between the muscle and the surrounding tissue, reoperations might be easier and the function of the muscle may be better preserved. MISS openings allow the practitioner to perform all types of strabismus surgeries, namely rectus muscle recessions, resections, plications, reoperations, retroequatorial myopexias, transpositions and oblique muscle recessions or plications.
\end{abstract}

\section{Keywords}

Strabismus surgery, conjunctiva, conjunctival opening, minimally invasive strabismus surgery (MISS), Parks' cul-de-sac approach

Disclosure: The author has no conflicts of interest to declare.

Received: 18 January 2011 Accepted: 20 March 2011 Citation: European Ophthalmic Review, 2011;5(1):27-32 DOI: 10.17925/EOR.2011.05.01.27

Correspondence: Daniel S Mojon, Department of Strabismology and Neuro-Ophthalmology, Kantonsspital St Gallen, 9008 St Gallen, Switzerland. E: daniel.mojon@kssg.

This article reviews minimally invasive approaches to strabismus surgery, which reduce tissue trauma, post-operative patient discomfort, hospital stay and working disability. For such techniques, it is advisable to use the operating microscope, although they can also be performed using magnifying spectacles. Usually, a higher magnification allows a less traumatic tissue dissection and a better control of bleeding. Chemodenervation therapy, which can also be considered a minimally invasive procedure, will not be discussed here. Even if this seems obvious, it should be mentioned that strabismus surgery is indicated only after considering all non-surgical options and if appropriate, after they have been tried. A non-surgical treatment remains less invasive than the least invasive surgical procedure.

The post-operative visibility of the procedure, patient discomfort, hospital stay and working disability will be mainly influenced by the number and type of muscles operated on and the type and location of the conjunctival openings. For rectus muscles, the majority of strabismus surgeons use Harms' limbal approach, ${ }^{1}$ which has been popularised by von Noorden. ${ }^{2.3}$ This is a limbal opening over a quadrant, allowing full visualisation of the operated muscle. Since one cut is performed along the limbus, patients usually experience an increased post-operative discomfort and the interpalpebral conjunctiva will be red in the first couple of weeks. Such an opening is also prone for corneal dellen formation and Tenon's prolapse. In order to avoid these disadvantages, Parks introduced and popularised a fornix-based conjunctival incision for rectus muscle access, which remains covered by the lids after surgery. ${ }^{4}$ The technique is elegant, however, its use is difficult in older patients with inelastic conjunctiva, in young patients with prominent Tenon's tissue and in cases with significant pre-existing scarring. There is also the need for an assistant surgeon to adequately visualise the operating site and the anatomical disruption between the muscle and perimuscular tissue is not significantly decreased compared to Harms' opening. Gobin was the first to describe the principle of access for rectus muscles through two small radial openings - one along the superior and the other along the inferior muscle margin. ${ }^{5-7}$ He showed that rectus muscle loop recessions could be performed through such openings. This principle of access has been adapted in order to be able to perform all types of strabismus surgeries, namely rectus muscle recessions, resections, plications, reoperations, retroequatorial myopexias and transpositions and oblique muscle recessions or plications.

\section{Minimally Invasive Strabismus Surgery}

The concept of minimally invasive strabismus surgery (MISS) consists of the following principles:

- placement of all conjunctival cuts as far away from the limbus as possible;

- avoidance of conjunctival opening where not necessary to perform the surgical steps;

- reduction of total conjunctival opening size by using several keyhole openings instead of one large access;

- placement of keyhole cuts in a way to permit joining them if increased visibility is needed; 


\section{Surgery}

Figure 1: Minimally Invasive Strabismus Surgery Rectus Muscle Recession and Plication
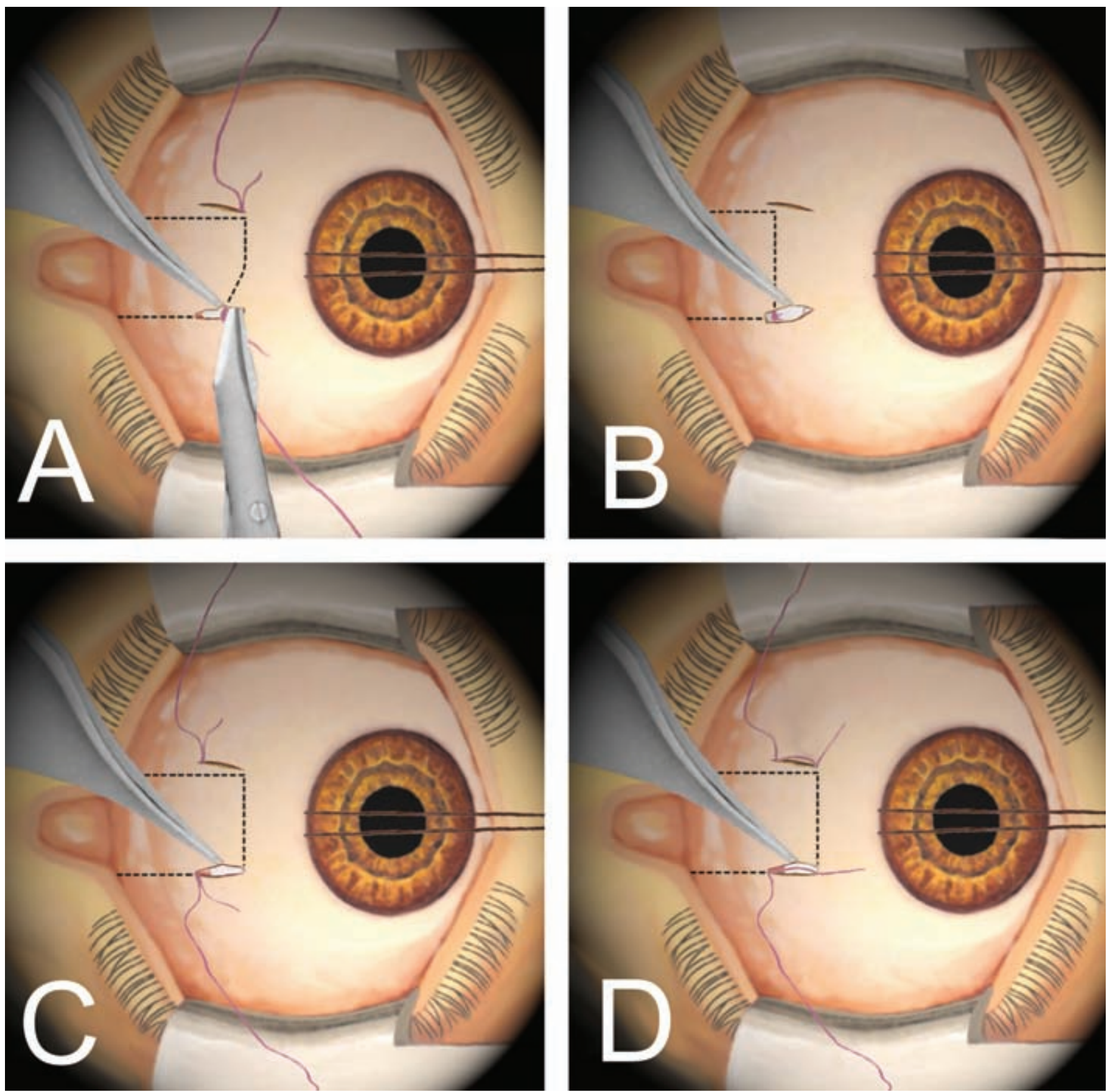

Minimally invasive strabismus surgery (MISS) recession: after suturing the muscle insertion the muscle is disinserted (A). The muscle is reattached after measuring the recession distance with a calipter (B). ${ }^{8}$ MISS marginal dissection (MADI) plication: after measuring the plication distance with a caliper, two sutures are applied to the muscle borders (C). After passing through the original insertion, the muscle is plicated by pulling the two sutures without using a spatula (D).

- performance of all feasible surgical steps through tunnels; and

- minimisation of perimuscular tissue disruption.

Instead of one large opening, several keyhole cuts are placed where the main surgical steps - usually suturing - have to be performed. If necessary, for some surgical steps tunnels are created between the cuts. The openings are always placed far away from the limbus in a way that, if necessary, a usual, large opening can be achieved by joining them. For example, for rectus surgery, the two parainsertional cuts can be prolonged anteriorly and joined at the limbus in order to achieve Harms' opening. Perimuscular tissue disruption is always minimised. For strabismus surgeries following these principles, the term MISS has been introduced. ${ }^{6,8}$ Post-operatively, MISS openings will remain covered by the eyelids and will minimise visibility of surgery, patient discomfort and limbus opening-related complications, for example, corneal complications. Long-term benefits include avoidance of an increase of redness of the visible conjunctiva and a decreased scarring of the perimuscular tissue, which will facilitate reoperations.

There is increasing evidence suggesting that the disruption of the perilimbal episcleral vessels - which occurs with a limbal incision may predispose to anterior segment ischaemia. ${ }^{9}$ MISS will preserve the majority of perilimbal episcleral vessels. Since MISS also 

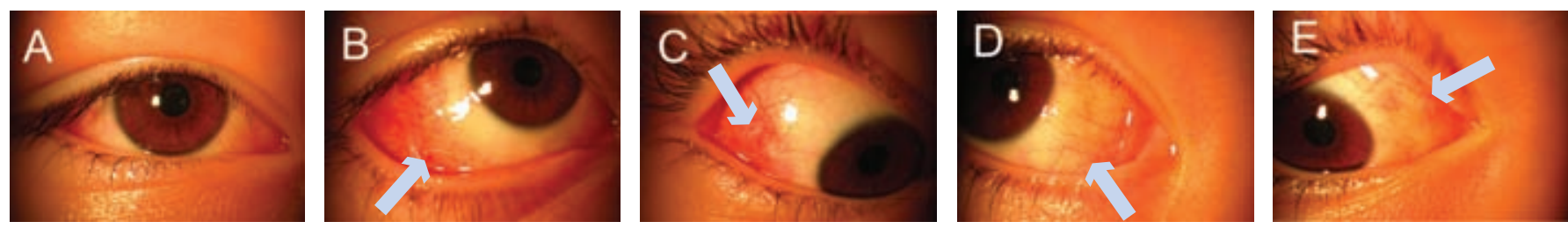

Photographs showing appearance of the right eye of a 10-year-old patient on the first post-operative day after $4.5 \mathrm{~mm}$ lateral rectus muscle recession and $4 \mathrm{~mm}$ medial rectus muscle plication. Eye in primary position with all surgical openings covered by the lids (A). Conjunctival openings are only visible in extreme gaze positions. Arrows mark the small cuts near the insertion of the medial and lateral rectus muscle $(B-E)$.

significantly reduces the anatomical disruption between the muscle and the surrounding tissue, reoperations might become easier and the function of the muscle might possibly be preserved better.

\section{Muscle Exposure for Minimally Invasive Strabismus Surgery}

An optimal exposure of the operated muscle can be achieved by one silk 6-0 limbal traction suture. Such a suture allows to perform all MISS surgeries without an assistant surgeon. Rarely, the scrub nurse may need to briefly hold a spatula to better visualise the operating field, for example in superior oblique surgery. Direct contact of the traction suture with the cornea must be avoided because of the high risk of corneal erosion. Although some surgeons performing MISS do not close the keyhole cuts at the end of surgery, we always close each cut with one or more single, resorbable 8-0 or 9-0 sutures, because the risk of post-operative infection might otherwise be increased. Since the cuts are far away from the limbus, usually this will not induce a foreign body sensation.

\section{Minimally Invasive Strabismus Surgery for Rectus Muscle Recession and Plication}

Two small radial keyhole parainsertional openings are performed..$^{6,8,10}$ For correct cut placement, identify the muscle vessels through the conjunctiva. Patients with reduced elasticity of the conjunctival tissue require larger cuts in order to avoid conjunctival tearing while working with instruments. After identifying the borders of the rectus muscle, it is hooked. A tunnel is created between the two cuts. To perform a recession, two sutures are applied at the lateral borders of the rectus muscle tendon as close as possible to the insertion. If prominent vessels are visible at the insertion site, they should be cauterised. The tendon is then detached using a Wescott scissor (see Figure 1A). After measuring the amount of recession, the tendon is reattached with the two sutures to the sclera (see Figure 1B). Alternatively, the muscle reattachment sutures can be prepared before disinserting the muscle. Recently, for larger recessions, a MISS transconjunctival suturing technique (TRASU) has been developed. ${ }^{11}$ It allows for the reattaching of a muscle far behind the original insertion without the need to prolong the cut more posteriorly. To perform a rectus muscle plication, the same access is chosen. With the original MISS plication technique, a tunnel was created before plication. ${ }^{8}$ However, it has been shown that this is not necessary. A marginal dissection (MADI) of the rectus muscle is sufficient. ${ }^{12}$ After identifying the borders of the muscle, it is hooked. Two sutures are applied to the upper and lower borders of the muscle at the distance from the tendon insertion site corresponding to the plication amount (see Figure $1 \mathrm{C}$ ). If at this location prominent vessels are visible, they should be cauterised before suturing. Next, the sutures are passed at the superior and inferior tendon insertions (see Figure 1D). The muscle is plicated by tying the two sutures. Usually, on the first post-operative day, in the primary gaze position, surgery is hardly visible. The cuts can be visualised on side gaze by pulling on the eyelids. Figure 2 shows a patient on the first post-operative day after horizontal rectus muscle recession and plication surgery.

\section{Minimally Invasive Strabismus Surgery for} Rectus Muscle Posterior Fixation Suture

For access, two small, L-shaped cuts are performed slightly anterior to the location where the scleromuscular sutures will be placed. A more anterior prolongation of the cuts will allow combining retroequatorial suturing with a MISS recession or plication. Using blunt Westcott scissors, the episcleral tissue is separated from the muscle sheath and the sclera. Then, a measure caliper is used to determine the exact placement of the scleromuscular sutures. The posterior fixation is done by passing a non-resorbable suture through the sclera, followed by the muscle suture, which will include approximately one-third of both lateral parts of the muscle (see Figure $3 A)$. Tightening is performed by a three-throw adaptation suture followed by two securing loops. ${ }^{13}$

\section{Minimally Invasive Strabismus Surgery for Rectus Muscle Transposition}

For a partial muscle transposition of both adjacent muscles, four openings will suffice. For complete transposition, six openings are necessary. The half-muscle that will be transposed is exposed by a small, radial cut. Using blunt Wescott scissors, the episcleral tissue is separated from the muscle sheath and the sclera. Then, the muscle is hooked. Now, on the side of the opening, a meticulous dissection of the check ligaments and intermuscular membrane is performed $7 \mathrm{~mm}$ backward to the insertion. The resulting tunnel will allow for the easy splitting of the muscle. A curved ruler is used to measure the muscle insertion width and to determine its midpoint. Starting at the midpoint, the muscle is gently split using a small muscle hook with a mark $15 \mathrm{~mm}$ away from the hook. Splitting is stopped when the mark reaches the insertion. Two single sutures are applied to the muscle tendon, which will be transposed. Using a Wescott scissor, the splitted half-muscle is detached from the insertion. The same technique is used to split the other rectus muscle. The minimally invasive transposition is performed by exposing the weak rectus muscle through two small parainsertional keyhole cuts. Now, a blunt 20G sub-Tenon's anaesthesia cannula is inserted through one of these openings and advanced in order to get out through the contralateral cut, safely displacing the two needles and the half-muscle (see Figure 3B). If additional force is generated after undercrossing the weak muscle, the half-muscle is reattached. The same method is performed with the second half-muscle. Full-tendon transposition can also be performed using six keyhole openings. ${ }^{14}$ Figure 4 shows a seven-year-old patient after transposition of half of the medial and lateral rectus muscle to the superior rectus muscle. On the first post-operative day, the perilimbal region is only mildly red. Since MISS 


\section{Surgery}
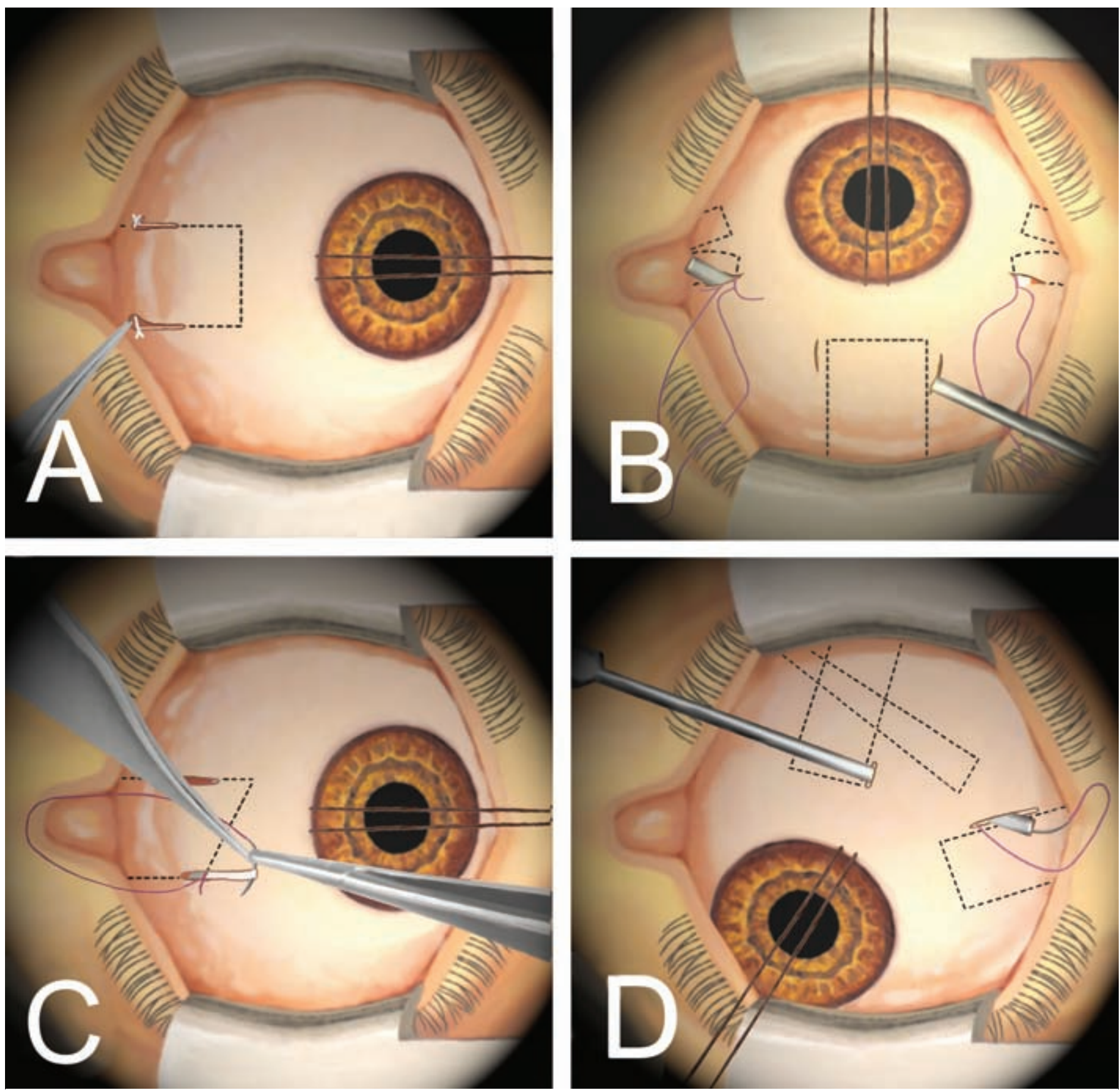

Minimally invasive strabismus surgery (MISS) rectus muscle retroequatorial myopexia (A); ${ }^{13}$ MISS partial rectus muscle transposition (B); ${ }^{14}$ MISS rectus muscle repeat surgery, here a muscle advancement (C); 15 MISS graded inferior oblique recession (D). 16

transpositions are possible, simultaneous bilateral transpositions can also be performed.

Minimally Invasive Strabismus Surgery for Rectus Muscle Repeat Surgery

The principle of MISS surgery can also be applied to repeat rectus muscle surgery. Knowledge of what surgery has been done previously will allow minimising of the MISS keyhole openings by placing them exactly where the muscle insertions lie. Alternatively, the insertion can be determined pre-operatively, using the slit-lamp, or intra-operatively, by observation of the movement of the vessels while the eye moves. Conjunctival vessels will freely rotate, while the vessel at the insertion will not. The big advantage of a MISS approach is that the surgeon will not have to reopen an already traumatised perilimbal conjunctiva and thus, avoids the risk of a permanent increase of conjunctival redness and scarring and probably also decreases the risk of anterior segment ischaemia.?

The principals of surgery are similar to primary MISS rectus recessions and plications. Previously recessed muscles should be advanced and not plicated ${ }^{15}$ (see Figure 3C).

Minimally Invasive Strabismus Surgery for Inferior Oblique Recession

MISS inferior and superior oblique recessions of more than $6 \mathrm{~mm}$ are performed using two keyhole openings. Through the first one, the disinsertion is performed and a second one is used for scleral anchoring. In order to safely pass the needle between the two cuts, a 
blunt 20G sub-Tenon's anaesthesia cannula is used (see Figure 3D). ${ }^{16}$ MISS inferior and superior oblique muscle plications are done through one L-shaped opening over the insertion. ${ }^{6}$ Figure 5 shows a patient on the first post-operative day after graded inferior oblique recession.

\section{Minimally Invasive Strabismus Surgery for} Surgery of Fibrotic Rectus Muscles

In non-fibrotic muscles there are two reasons why the scleral sutures at the new insertion site should be prepared before detaching the insertion: first, a still-attached rectus muscle will ensure that the muscle is precisely displaced along its normal path; second, a bleeding occurring after muscle disinsertion will make scleral suturing more demanding. In fibrotic muscles - like in Duane retraction syndrome or thyroid eye disease - scleral sutures cannot be applied before the eye has been turned away from the field of surgery. Therefore, in such cases, scleral anchoring at the new site can only be performed after muscle disinsertion. ${ }^{6}$

\section{Suggestions for How to Start Doing Minimally Invasive Strabismus Surgery}

The use of a microscope for MISS is advocated. Surgeons switching from magnifying glasses to the operating microscope should first perform several procedures with their own technique under the microscope before starting with MISS. Start with primary horizontal rectus muscle recessions and plications of $4 \mathrm{~mm}$ or less in patients with aged between 14 and 40 years. The abundant Tenon's tissue will make surgery more difficult in very young patients and reduced elasticity of the conjunctiva increases the risk of a conjunctival tear in older patients. ${ }^{6}$ The ideal practice before starting with a new technique is to personally visit a surgeon using it on a regular base. I always welcome colleagues who want to come and assist MISS.

\section{Instruments Suitable for Minimally Invasive Strabismus Surgery}

While instrument size in open surgery is not usually critical, conjunctival tearing will occur if the instruments used to operate through MISS keyhole openings are too large. The following instruments are useful to perform MISS. By clamping a serrefine to the eyelid speculum, a hypomochlion is created for traction sutures applied medially. This will prevent corneal rubbing of the traction suture. A colibri forceps with interdigitating teeth is used to fix the conjunctiva while performing the keyhole openings and to stabilise the eye for traction and scleral anchoring sutures. A small, curved needle holder is optimal to perform suturing through small openings. A small conjunctival scissor with a curved, blunt tip is used to cut the conjunctiva, the sutures and the muscle or tendon insertions. Curved forceps with serrated tips should be used to hold the conjunctiva after performing the keyhole openings since this will prevent unnecessary conjunctival trauma. Spatulas of different sizes are useful to visualise the tissue through the small, conjunctival cuts. Diathermy is best performed using a system with a bipolar, coaxial tip. This will allow gentle cauterisation of vessels at the muscle insertion through the tunnel without damaging the overlying conjunctiva. A small strabismus hook will allow other instruments to be introduced through the keyhole cuts without tearing the conjunctiva. Angled forceps are necessary to stabilise the muscle insertion during disinsertion. By pressing the blunt tips of a Castroviejo caliper for about ten seconds against the sclera, a bluish indentation mark is formed, which will be visible for up to one minute. This indentation technique also works transconjunctivally, since there is no need to
Figure 4: Post-operative Appearance after Minimally Invasive Strabismus Surgery Rectus Muscle Transposition

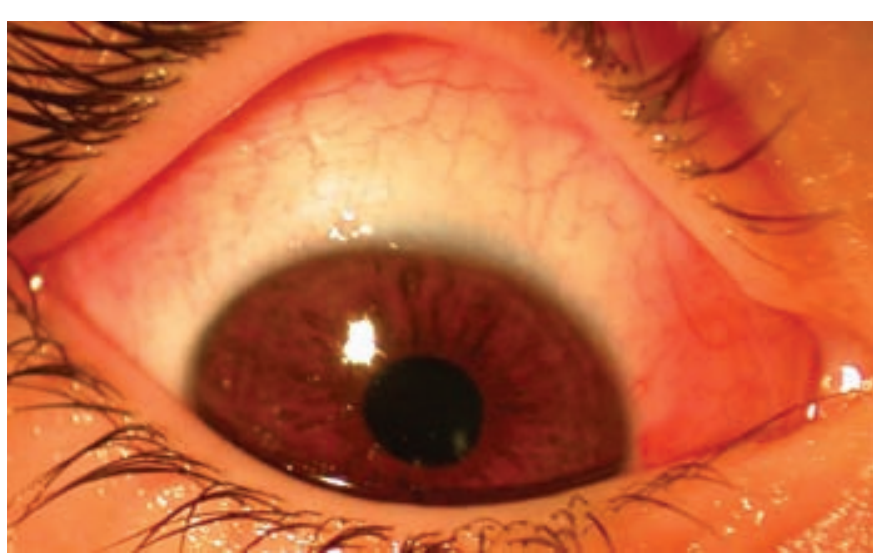

In this seven-year-old patient, half of the medial and lateral rectus muscle were transposed to the superior rectus muscle. On the first post-operative day, the perilimbal region is only mildly red.

Figure 5: Post-operative Appearance after Minimally Invasive Strabismus Surgery Inferior Oblique Surgery

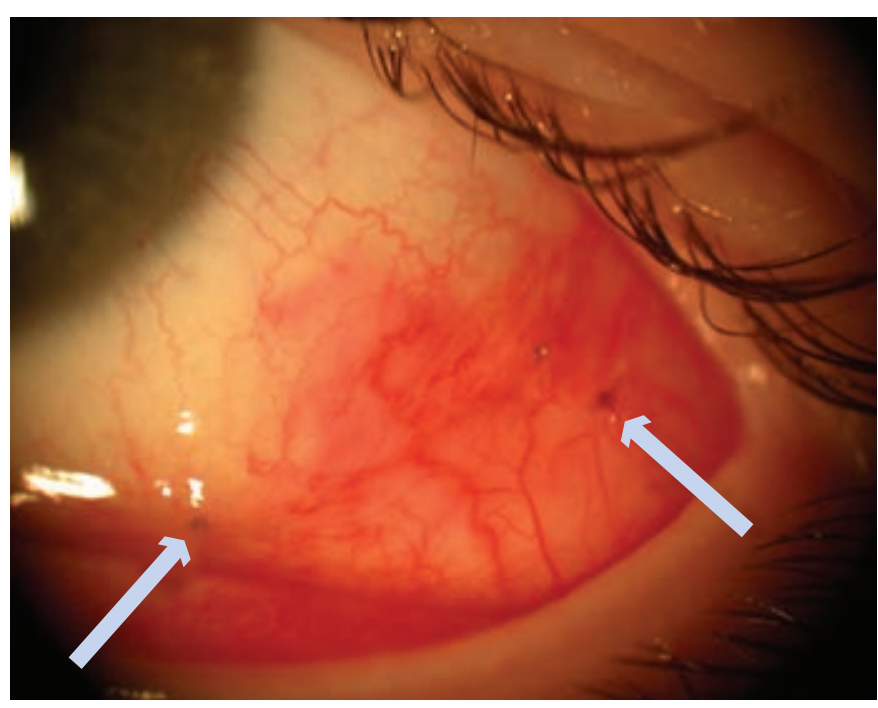

Appearance of the eye on the first post-operative day after graded inferior oblique recession on upgaze after pulling away the inferior lid. The two keyhole openings are visible.

apply a dye to the tip. A blunt cannula is helpful to safely displace needles through the tunnel between two key openings. ${ }^{6}$

\section{Minimally Invasive Strabismus Surgery Dose-response Relationships}

If MISS muscle displacements are performed in the same way as in open surgery, it seems rather unlikely that dose-response relationships will differ after switching operating technique. If a surgeon modifies one or more steps while changing to MISS, there will be a risk of a systematic under- or overcorrection. As a result, it is prudent if surgeons switching to MISS look for changes in their dose-response relationships. ${ }^{6}$

\section{Conclusions and Future Directions}

In conclusion, placing several keyhole openings far away from the limbus instead of using one, large limbal opening, will dramatically reduce the surgical trauma. This will also improve the quality of life of the operated patients in the direct post-operative period. In contrast to Parks' technique, MISS can also be easily used in patients 
with highly inelastic conjunctiva, for example, in old patients or patients with thyroid endocrine orbitopathy. Compared to Harms' limbal opening, Parks' opening reduces tissue trauma. MISS allows a further reduction by minimising the perimuscular tissue dissection. One disadvantage of Parks' technique and MISS is that no postoperative adjustment is possible. Therefore, we developed a modification of the MISS technique to adjust to a certain degree, the rectus muscles on the first post-operative day. We hope that the number of surgeries performed with this technique will soon determine whether the new technique is adjustable enough to be popularised. What else can we expect in the future? We are developing instruments specifically designed for the small MISS openings. Such instruments should allow muscle displacements to be performed faster and with less trauma.
1. Harms $\mathrm{H}$, Über Muskelvorlagerung, Klin Monatsbl Augenheilk, 1949:115;319-24.

2. von Noorden GK, The limbal approach to surgery of the rectus muscles, Arch Ophthalmol, 1968;80:94-7.

3. von Noorden GK, Modification of the limbal approach to surgery of the rectus muscles, Arch Ophthalmol, 1969;82:349-50.

4. Parks MP, Fornix incision for horizontal rectus muscle surgery, Am I Ophthalmol, 1968;65:907-15.

5. Gobin MH, Bierlaagh JJM, Simultaneous Horizontal and Cyclovertica Strabismus Surgery, Antwerp: Centrum voor Strabologie, 1994.

6. Mojon DS, Minimally invasive strabismus surgery. In: Fine HI, Mojon DS (eds), Minimally Invasive Ophthalmic Surgery, US: Springer, 2009;123-52

7. Mojon DS, Minimally invasive strabismus surgery,
BrJ Ophthalmol, 2009:93:843-4.

Mojon DS, Comparison of a new, minimally invasive strabismus surgery technique with the usual limbal approach for rectus muscle recession and plication, Br J Ophthalmol, 2007;91:76-82.

9. Kushner BJ, Comparison of a new, minimally invasive strabismus surgery technique with the usual limbal approach for rectus muscle recession and plication, Br $\perp$ Ophthalmol, 2007:91:5.

10. Pellanda N, Mojon DS, Minimally invasive strabismus surgery technique in horizontal rectus muscle surgery for esotropia, technique in horizontal rectus
Ophthalmologica, 2010;224:67-71.

11. Mojon DS, A New Transconjunctival Muscle Reinsertion Technique for Minimally Invasive Strabismus Surgery, J Pediatr Ophthalmol Strabismus, 2009;2:1-5.
12. Moion DS, A Modified Technique for Rectus Muscle Plication in Minimally Invasive Strabismus Surgery, Ophthalmologica, 2009;224:236-42.

13. Mojon DS, Minimally invasive strabismus surgery for rectus muscle posterior fixation, ophthalmologica, 2009;223:111-5

14. Mojon DS, Minimally Invasive Strabismus Surgery (MISS) for Rectus Muscle Transpositions, $\mathrm{Br}$ I Ophthalmol, 2009:93:747-53.

15. Mojon DS, Minimally invasive strabismus surgery for horizontal rectus muscle reoperations, Br 1 Ophthalmol, horizontal rectus mu

16. Mojon DS, Minimally invasive strabismus surgery (MISS) for inferior obliquus recession, Graefes Arch Clin Exp Ophthalmol, 2009;247:261-5 